Geografia e Ordenamento do Território, Revista Eletrónica

Centro de Estudos de Geografia e Ordenamento do Território

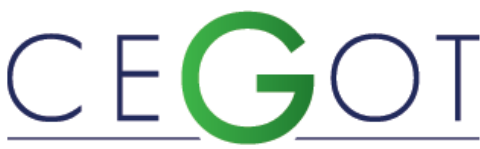

Centro de Estudos de Geografia e Ordenamento do Território http://cegot.org

ISSN: 2182-1267

Laranjeira, M.

Departamento de Geografia, Universidade do Minho Centro de Estudos Geográficos, Universidade de Lisboa

manuela.laranjeira@geografia.uminho.pt

\title{
Estrutura espacial e processos ecológicos: o estudo da fragmentação dos habitats
}

Referência: Laranjeira, M. (2012). Estrutura Espacial e Processos Ecológicos: O estudo da Fragmentação dos Habitats. Revista de Geografia e Ordenamento do Território, n.o 1 (Junho). Centro de Estudos de Geografia e Ordenamento do Território. Pág. 59 a 83

\section{Resumo}

O presente artigo revê a relação entre alguns conceitos e pressupostos fundamentais para a atual investigação ecológica e biogeográfica que, desde meados de 1980, se tem vindo a centrar no estudo do regime e dos efeitos das perturbações ambientais, naturais e antrópicas, sobre espécies e comunidades biológicas. Salienta-se a afirmação crescente da Ecologia da Paisagem, disciplina que enfatiza a importância da heterogeneidade espacial e das relações recíprocas entre os padrões espaciais da paisagem e os processos ecológicos, sobretudo no contexto de uma perturbação como a da fragmentação dos habitats. A base conceptual e os resultados dos principais 
modelos de simulação espacial e dos estudos empíricos que permitem destrinçar a importância da perda do habitat relativamente à configuração espacial do habitat são sintetizados e discutidos.

Palavras-Chave: Ecologia da Paisagem, Biogeografia, perturbação ambiental, fragmentação do habitat, perda do habitat, conectividade do habitat, metapopulação

\section{Abstract}

This paper reviews the relationship between concepts and assumptions most relevant to contemporary ecological and biogeographical research, which became focused on the study of environmental disturbance regimes and effects on species and biological communities since the mid-1980s. Landscape ecology, with its rising status, emphasizes spatial heterogeneity and the reciprocal relations between landscape spatial patterns and ecological processes, particularly in the context of habitat fragmentation. The conceptual basis and results from the major simulation and empirical studies to date that highlight the relative importance and effects of habitat loss and habitat spatial configuration are synthesized and discussed.

Keywords: Landscape Ecology, Biogeography, environmental disturbance, habitat fragmentation, habitat loss, habitat connectivity, metapopulation 


\section{Introdução}

\subsection{Questões antigas, novas abordagens}

Uma revisão dos artigos publicados, entre 1904 e 2003, na revista 'Annals of the Association of American Geographers' (que representa uma amostra importante da investigação geográfica) nota que, da investigação em Biogeografia, desde o início do século passado, 4 temáticas se destacam como recorrentes, ainda que a proeminência e a relevância atribuída a cada uma delas tenham variado consideravelmente entre os diferentes períodos epistemológicos atravessados: (i) padrões espaciais e processos ecológicos; (ii) evolução da paisagem; (iii) modificação das comunidades biológicas pela ação antrópica; e, (iv) interligação dos sistemas físicos e biológicos (Cowell e Parker, 2004). Não obstante a sua recorrência, a abordagem conceptual e metodológica às questões nucleares para a Biogeografia evoluiu a par dos principais paradigmas da Geografia Física, da Biologia e da Ecologia. Assim, a partir de meados da década de 1980, o estudo do regime das perturbações ambientais e da resposta diferencial das espécies a estas perturbações tem ocupado um lugar central na investigação biogeográfica (Cowell e Parker, 2004), em paralelo com a nova ênfase dada à análise espacial pelos ecologistas e a rápida afirmação da Ecologia da Paisagem (Kent, 2007; 2009).

A Ecologia da Paisagem - termo introduzido por Carl Troll, em 1939, e retomado pelo geógrafo alemão em 1950 (Turner, 2005) - surgiu da tradição europeia em estudos relacionados com a geografia regional e a vegetação (Turner, 1989), e teve por impulso a nova perspetiva de investigação oferecida pela fotografia aérea (Turner, 2005). Todavia, esta disciplina só experimentou um real desenvolvimento desde 1985 (Li e Wu, 2004; Turner, 2005), à medida que a gravidade dos impactes ambientais, resultantes das extensas e profundas transformações do uso do solo, se foi tornando uma evidência incontornável. Por sua vez, Fortin et al. (2003) dão conta de como a rápida evolução tecnológica associada aos sistemas de informação geográfica foi motivada e, simultaneamente, estimulou o recente progresso da pesquisa ecológica, em particular da Ecologia da Paisagem. Em estreita relação com a escala espacial (e temporal) de análise permitida pelos dados com origem nos sistemas de deteção 
remota, a paisagem é conceptualizada, no âmbito da disciplina, como um "mosaico ecológico" (Forman, 1995) - i.e., uma área espacialmente heterogénea (Turner, 1989; 2005), caracterizada (i) pela composição (diversidade) e estrutura, ou configuração espacial, dos seus elementos constituintes (habitats), (Gustafson, 1998), (ii) pela dinâmica funcional (processos) entre os seus elementos espaciais (fluxos de energia, matéria e organismos), e, por fim, (iii) pela sua evolução no tempo (alterações na composição, estrutura espacial e processos ecológicos), (cf. Turner, 1989). A temática de interesse da Ecologia da Paisagem tem-se, pois, centrado nas interações recíprocas entre padrões espaciais e processos ecológicos, escala e hierarquia ( $\mathrm{Li} \mathrm{e} \mathrm{Wu,} \mathrm{2004),}$ dando particular atenção à forma como a estrutura espacial da paisagem influencia a abundância e a distribuição dos organismos (Fahrig, 2005). Nesta sequência, foram desenvolvidos inúmeros índices de estrutura espacial, algoritmos largamente utilizados em Ecologia da Paisagem para a análise de padrões espaciais, sob a premissa de que podem funcionar como "elo de ligação" entre a estrutura espacial e os processos ecológicos (Gustafson, 1998). Porém, apesar do boom dos anos iniciais (1985-95), a visão atual sobre estes índices é bastante mais crítica, e revela até um certo ceticismo, sobretudo no que diz respeito ao modo como os resultados obtidos têm sido interpretados e utilizados em diversos estudos (e.g., ver sínteses críticas de Wu e Hobbs, 2002; Fortin et al., 2003; Li e Wu, 2004; Turner, 2005; e, recensão em Kent, 2009).

\subsection{Perturbação ambiental, desequilíbrio dos sistemas ecológicos e heterogeneidade espacial}

O paradigma da Ecologia e da Biogeografia contemporâneas rejeita a visão de que os sistemas ecológicos são inerentemente estáveis e sustenta que, na sua generalidade, estes não se encontram em equilíbrio dinâmico, mas em desequilíbrio (Perry, 2002; Rohde, 2005). Às perturbações ambientais reconhece-se cada vez mais a importância na regulação da estrutura e dinâmica não linear das comunidades biológicas (Jentsch, 2007). Neste contexto, considera-se uma perturbação ambiental qualquer fenómeno externo natural ou antrópico, espacial e temporalmente discreto (Łaska, 2001), que altera a estrutura de comunidades e populações de espécies, afeta a disponibilidade de recursos ou modifica as características físicas do ambiente (White e Pickett, 1985), 
desencadeando a sucessão ecológica (e.g., Walker e del Moral, 2003) e promovendo a heterogeneidade temporal e espacial dos habitats (Denslow, 1985; Gustafson e Diaz, 2002), numa mesma área geográfica. Com efeito, a dinâmica ecológica prende-se, em grande parte, com a interação entre as perturbações, a heterogeneidade espacial e a sucessão das comunidades, que é entendida como Bonan (2002) sintetiza: "Succession is a consequence of differential growth, survival, and colonizing ability of species [...] Individual species colonize where conditions are favorable, die out of the community when the environment is no longer favorable, and grow in company of other species with similar environmental requirements" (pp.437-438).

As teorias neorreducionistas da sucessão ecológica, que se acrescem às ideias individualistas de Gleason (Matthews, 1996) - a antítese das do seu contemporâneo Clements, percursor das teorias neo-holistas (ver excelente síntese em Walker e del Moral, 2003) -, enfatizam a responsabilidade das perturbações (e.g., Huston, 1994), dos eventos fortuitos, da história passada e da dispersão aleatória (e.g., Hubbell, 2001) sobre a composição das comunidades biológicas, defendendo que estas constituem grupos abertos de espécies, em contínua mudança ou desequilíbrio (Krebs, 2001). Assim, o estudo de certos atributos individuais das espécies, que determinam o tipo de resposta às alterações ambientais provocadas por uma perturbação, ganhou relevância na investigação ecológica, desde meados da década de 1980 (sendo que, para isso, foi essencial o contributo de Noble e Slatyer, 1980). Por sua vez, também o renovado interesse, a partir de 1985, na análise espacial (e temporal) da paisagem, tem as suas raízes em Gleason e nas teorias que prolongaram a sua obra. Com efeito, ao trabalhar preferencialmente a escalas espaciais pequenas, analisando intervalos de tempo longos (no âmbito da paleoecologia e da paleobiogeografia), tanto Gleason como sucessivos investigadores foram sendo confrontados com a espantosa heterogeneidade espacial e a efemeridade das comunidades de espécies (Hubbell, 2001), que consideraram incompatível com a visão Clementsiana. Com o progressivo desenvolvimento e a aceitação generalizada das ideias neorreducionistas (da década de 1970 em diante), operou-se a transição para o paradigma atual da Ecologia. Perry (2002) relaciona a mudança na forma como espaço e interações espaciais passaram a ser conceptualizados nas teorias ecológica e biogeográfica, bem como o interesse atual 
no estudo (teórico e empírico) da heterogeneidade espacial da paisagem, com esta transição paradigmática, relembrando: "Traditionally ecology has adopted a largely homogeneous view of space. Attention has been focused upon and within small areas [...] and it has been assumed that ecological interactions are spatially uniform. [...] Indeed, if ecosystems are perceived as homogeneous and their dynamics can be explained using equilibrium models then the explicit addition of spatial heterogeneity becomes problematic" (p.340).

Tendo em conta o papel fundamental das perturbações e da heterogeneidade espacial (logo, da estrutura da paisagem) na dinâmica ecológica, Woodley e Theberge (1992) definem integridade ecológica não como um estado normal ou final, de equilíbrio estável - tal como é comum noutros autores (e.g., Karr, 1997; Feinsinger, 2001) que, implicitamente, apelam ao conceito de comunidade clímax ${ }^{1}$ e retêm, desta maneira, a perspetiva determinista e unidirecional das ideias de Clements sobre a sucessão ecológica (datadas de 1910-30 mas fortemente enraizadas no pensamento ecológico e biogeográfico) -, e sim como um estado ótimo na evolução dos sistemas ecológicos, em função da história das pressões antrópicas a que foram sujeitos e do seu contexto espacial. De facto, a paisagem foi, na maioria das vezes, drasticamente moldada pela intervenção humana (e.g., August et al., 2002), pelo que a integridade de habitats, comunidades e espécies será necessariamente, afetada - senão mesmo determinada , pelo grau de transformação e de degradação do mosaico ecológico em que se inserem. Janzen, já em 1986, chamou a atenção para este problema, no âmbito da conservação em áreas protegidas, expressando-o eloquentemente como "the eternal external threat". Por sua vez, a importância do contexto espacial dos habitats inteiramente dependente da escala de análise e da forma como se configura a paisagem - é uma questão que, tal como argumenta Wiens (2002), define a própria essência da Ecologia da Paisagem.

\footnotetext{
${ }^{1}$ Por comunidade clímax entende-se a comunidade final da sucessão ecológica, para a qual todas as comunidades de uma área geográfica, com determinadas condições ambientais, evoluem de forma convergente (unidirecional), em equilíbrio estável com os factores abióticos e bióticos, e que se autoperpetua.
} 


\section{Relação entre Estrutura Espacial e Processos Ecológicos: a Fragmentação do Habitat}

\subsection{Perda da biodiversidade e a fragmentação dos habitats}

Dias (2001) classifica o fenómeno da perda da biodiversidade como: (i) a perda da variabilidade genética, ou redução populacional; (ii) a perda da variabilidade de espécies, ou redução das comunidades, incluindo a diminuição da heterogeneidade ambiental dos habitats e o aumento do ratio entre espécies exóticas e nativas; (iii) a perda da variabilidade ecológica, ou perda de tipos funcionais de espécies, que controlam processos ecológicos vitais; e, ainda, (iv) a perda da variabilidade cultural e económica.

As principais causas apontadas para a perda da biodiversidade estão diretamente relacionadas com as perturbações de origem antrópica, nomeadamente: (i) a destruição (eliminação), a fragmentação e a degradação dos habitats (incluindo a poluição); (ii) a sobre-exploração de certas espécies ou comunidades biológicas; (iii) a introdução de espécies exóticas e a disseminação de doenças (Pité e Avelar, 1996; Meffe et al., 1997; Dias, 2001). Dentre estas, a fragmentação dos habitats é unanimemente considerada a causa mais generalizada da perda da biodiversidade, e aquela com os efeitos mais complexos (e.g., Forman, 1995; Meffe et al., 1997; Dias, 2001; Olff e Ritchie, 2002; Fischer e Lindenmayer, 2007; Laurance et al., 2011). Entende-se por fragmentação (cujas causas podem ser inúmeras) o processo pelo qual uma área relativamente extensa e contínua de um dado habitat é subdividida num certo número de fragmentos (ou manchas), com uma menor dimensão e separados entre si por uma matriz (em geral, usos do solo introduzidos) caracterizada por condições ambientais muito diferentes das do habitat original (cf. Forman, 1995). O processo de fragmentação conduz, pois, a uma importante modificação do padrão espacial do habitat, que resulta não só da perda da sua área total, como também de uma nova configuração espacial (Fahrig, 2003).

O reconhecimento da importância da fragmentação dos habitats pela comunidade científica tem-se traduzido no extraordinário avultar das publicações dedicadas à 
temática, desde 1980. Apenas para se ter uma ideia, a pesquisa feita a 26 de maio de 2012 na base 'ISI Web of Knowledge/ Web of Science', para o termo composto "habitat fragmentation", devolveu 8 entradas para o período de 1980-89, 644 para 1990-99, 3335 para 2000-09 e, finalmente, 1322 referências só em relação ao intervalo entre 2010 e maio de 2012. Além disso, não é surpreendente que, desta vastíssima investigação, uma grande parte se reúna em torno das consequências ecológicas da fragmentação dos habitats (e.g., ver resultados de 32 anos de investigação na Amazónia Central em Laurance et al., 2011), em particular dos seus efeitos sobre as espécies (excelentes revisões críticas a resultados empíricos são as de Fahrig, 2003; Henle et al., 2004; Ewers e Didham, 2006). Paradoxalmente, pouco comuns têm sido os estudos que se debruçam diretamente sobre duas questões de fundo. Primeira, em que condição a fragmentação do habitat constitui um fator suficientemente importante para, por si só, ter efeitos significativos sobre a biodiversidade? Segunda, dado que a redução da área inicial é inerente a qualquer modo de subdivisão do habitat, não será a fragmentação um conceito redundante com o da perda de habitat? Para Fahrig (2003), a asserção de que a fragmentação constitui algo mais do que a perda de habitat depende da existência de efeitos ecológicos que podem ser atribuídos a modificações no padrão espacial do habitat independentes da respetiva quantidade. Porém, a distinção entre os efeitos da perda de área (i.e., a redução da quantidade do habitat) e os da fragmentação stricto sensu (i.e., a mudança na configuração espacial do habitat remanescente) é complexa, uma vez que ambos os fenómenos espaciais exibem uma forte correlação (Smith et al., 2009). Assim, é fundamental (i) controlar as condições sob as quais se realizam os estudos empíricos (seja a proporção do habitat existente na paisagem ou o grau de fragmentação) situação que não é observada em muitos casos, como mostrou Fahrig (2003) -, e (ii) recorrer a métodos e medidas estatísticas adequadas para estimar o efeito ecológico relativo da área e da configuração espacial, tal como a regressão múltipla e os coeficientes parciais de regressão padronizados - ao contrário de outros métodos de acrescida complexidade, que procuram remover a colinearidade destes fatores espaciais (Smith et al., 2009). 


\subsection{Base conceptual: a teoria da biogeografia das ilhas e a teoria da metapopulação}

A interpretação mais comum para o declínio da biodiversidade resultante da fragmentação do habitat prende-se, por um lado, com a exclusão imediata de certas espécies devida à destruição parcial do habitat e, por outro, com o precipitar no tempo da extinção local de outras espécies (Collinge, 1996), associada ao reduzido tamanho das suas populações nos fragmentos menores de habitat (e.g., Henle et al., 2004; Ewers e Didham, 2006; Laurance et al., 2011). De facto, as populações pequenas são mais vulneráveis a processos estocásticos de carácter ambiental (nomeadamente, os relacionados com episódios extremos de certas perturbações naturais), demográfico e genético (Fischer e Lindenmayer, 2007), que facilmente as arrastam para uma situação em que o número de indivíduos passa a ser demasiado baixo para garantir o sucesso da reprodução e, portanto, a persistência da população - i.e., o número de indivíduos torna-se inferior ao tamanho mínimo viável da população (Pité e Avelar, 1996). O padrão "espécies-área" positivo tem sido documentado em diversos habitats, para diferentes taxas (Collinge, 1999; Laurance et al., 2002; 2011). Sendo uma relação conhecida de há muito (Arrhenius, 1921), os mecanismos que lhe estão na base foram avançados por MacArthur e Wilson na sua teoria da biogeografia das ilhas, ou teoria do equilíbrio dinâmico, de 1967 (citados em Pité e Avelar, 1996). Esta teoria preconiza que a diversidade de espécies, num dado local isolado de habitat, se trata de uma resultante entre as taxas de colonização e de extinção que variam, respetivamente, em função da proximidade desse local à fonte (origem) dos propágulos das espécies e da área do habitat. Assim, quanto menor for a área do habitat, mais elevada será a taxa de extinção local (porque haverá menos indivíduos por espécie) e menor o número de espécies presentes; por seu lado, o isolamento relativo do habitat contribui para agravar o efeito da área sobre a extinção local das espécies, uma vez que "as populações isoladas deixam de poder receber imigrantes de outros locais e nenhum processo se opõe ao seu declínio até à extinção" (Pité e Avelar, 1996, p.249).

A distribuição espacialmente disjunta das populações de diversas espécies é um resultado visível da subdivisão do respetivo habitat em vários fragmentos separados entre si. A persistência das espécies no habitat fragmentado, não obstante a extinção 
de algumas das suas subpopulações em manchas menores e mais isoladas, continua a verificar-se, pois "embora algumas [subpopulações] desapareçam, outras prosperam e produzem indivíduos que poderão recolonizar os locais onde ocorreu extinção" (Pité e Avelar, 1996, p.43). Esta dinâmica de regulação da população subdividida de uma dada espécie permite distinguir uma metapopulação, conceito introduzido por Levins, em 1969 (citado em Pité e Avelar, 1996), e que define um sistema de populações locais, ou subpopulações, interligadas por processos de extinções e recolonizações, que resultam na persistência do conjunto.

A teoria da metapopulação, impulsionada por Hanski e Gilpin (1997; citados em Thomas e Kunin, 1999), é atualmente uma base conceptual incontornável dos estudos sobre a dinâmica das populações em habitats espacialmente estruturados, como os habitats fragmentados ${ }^{2}$. De acordo com a teoria, a dispersão de uma espécie, a partir de um ou mais fragmentos (e subpopulações de origem), é fundamental para a ocorrência dessa mesma espécie noutras manchas do mosaico ecológico, que deixaram de reunir todas as condições favoráveis à sua presença, ao assegurar que a recolonização contrabalança a extinção da população local. Esta dinâmica espacial, em que a recolonização compensa o efeito da área sobre a extinção de uma subpopulação, é conhecida por "rescue-effect" ou processo de renovação, proposto em 1977 por Brown e Kodric-Brown (citados em Pité e Avelar, 1996; ver também "mass-effects", em Leibold et al., 2004). No caso dos habitats fragmentados, não é só a distância absoluta entre as manchas remanescentes que dificulta a dispersão das espécies entre elas e determina o seu grau de isolamento. A barreira, que constituem as novas margens dos habitats subdivididos e as características da matriz, pode ser tanto ou mais importante do que a configuração espacial para a ocorrência e o sucesso da dispersão (Ewers e Didham, 2006; Fahrig, 2007). Nesta sequência, o isolamento de uma mancha pode ser entendido como a sua inacessibilidade (Bender et al., 2003) em relação à dispersão dos animais e dos diferentes tipos de propágulos (i.e., sementes, rizomas horizontais, pólen), através dos quais se dá a dispersão das plantas; deste modo, ao maior isolamento da mancha corresponderá, teoricamente, a abundância

\footnotetext{
${ }^{2}$ Ver Leibold et al. (2004) para a discussão do conceito de metacomunidade.
} 
inferior ou a ausência das espécies que são incapazes de atingir, e colonizar, com sucesso a mancha em causa.

\subsection{Influência da configuração espacial do habitat}

Na recensão que se segue, deu-se particular relevo às conclusões dos trabalhos que procuraram responder diretamente às duas questões levantadas na secção 2.1. deste artigo. Os resultados dos inúmeros estudos empíricos, existentes até à data, não são convergentes quanto à importância relativa da perda de área e da configuração espacial dos habitats fragmentados sobre as populações e as comunidades de espécies analisadas; no entanto, é plausível que tal se possa dever ao facto de, por um lado, estas poderem manifestar uma resposta muito variável à estrutura espacial (tal como sucede relativamente a outros fatores abióticos e bióticos) e, por outro lado, a proporção do habitat remanescente na paisagem e o respetivo grau de fragmentação poderem ser muito diferentes entre os diversos estudos realizados, sendo assim difícil estabelecer generalizações. Posto isto, para a revisão da presente secção, salientou-se o contributo da investigação desenvolvida com base em modelos teóricos de simulação espacial, em que as condições de partida são perfeitamente controladas.

Mclntyre e Hobbs (1999) propuseram uma tipologia conceptual para diferentes estados de modificação da paisagem, em função do grau de destruição (i.e., perda de área total) do habitat (Fig.1). Os autores argumentam que ambos os processos da destruição e da modificação das características ecológicas do habitat podem ser conceptualmente abordados como um continuum, existindo várias combinações possíveis entre os dois tipos de gradiente. Segundo este modelo, uma paisagem fragmentada será aquela onde, devido à ocorrência de uma significativa perda do habitat - de que permanece, apenas, uma área relativa na ordem de $10-60 \%$-, a matriz corresponde já ao 'habitat destruído' e o que resta do habitat original encontrase circunscrito a um conjunto de manchas dispersas, por comparação com as paisagens intactas e variegadas (Fig.1). A diferenciação entre uma paisagem fragmentada e outra variegada é, no entanto, essencialmente funcional, e prende-se com a conectividade do habitat remanescente: "A functional distinction between variegated and fragmented landscapes is supported by theoretical landscape models that indicate organisms are operationally unfragmented when there is $>60 \%$ habitat 
retention [...] Between $10 \%$ and $60 \%$ retention (fragmented landscapes), the degree of fragmentation is highly dependent on the mobility of the organism and the arrangement of the habitat" (McIntyre e Hobbs, 1999, p.1285).

Os graus de modificação do habitat remanescente refletem, no modelo conceptual considerado, a tendência geral para este se tornar progressivamente mais degradado com o aumento da perda de área dos fragmentos, à medida que uma maior proporção da respetiva superfície vai ficando mais exposta à influência da matriz - i.e., o ratio em cada mancha entre o habitat da margem ('modificado') e o habitat interior ('não modificado'), vai sendo sucessivamente mais elevado (Fig.1). Porém, o modo como se processa a perda do habitat também influencia o grau de modificação ecológica, uma vez que vai ocasionar diferenças consideráveis no que respeita ao número, à dimensão, à forma (e.g., ratio entre perímetro e área) dos fragmentos e à distância entre eles (Fig.2); consequentemente, apesar da proporção do habitat restante poder ser equiparável, diferentes tipos de padrão espacial irão, em teoria, acarretar consequências ambientais, e efeitos sobre as espécies e as comunidades biológicas, sensivelmente distintos.

Figura 1 - Modelo conceptual dos estados de modificação de uma paisagem, segundo Mclntyre e Hobbs (adaptado de Fischer e Lindenmayer, 2007).

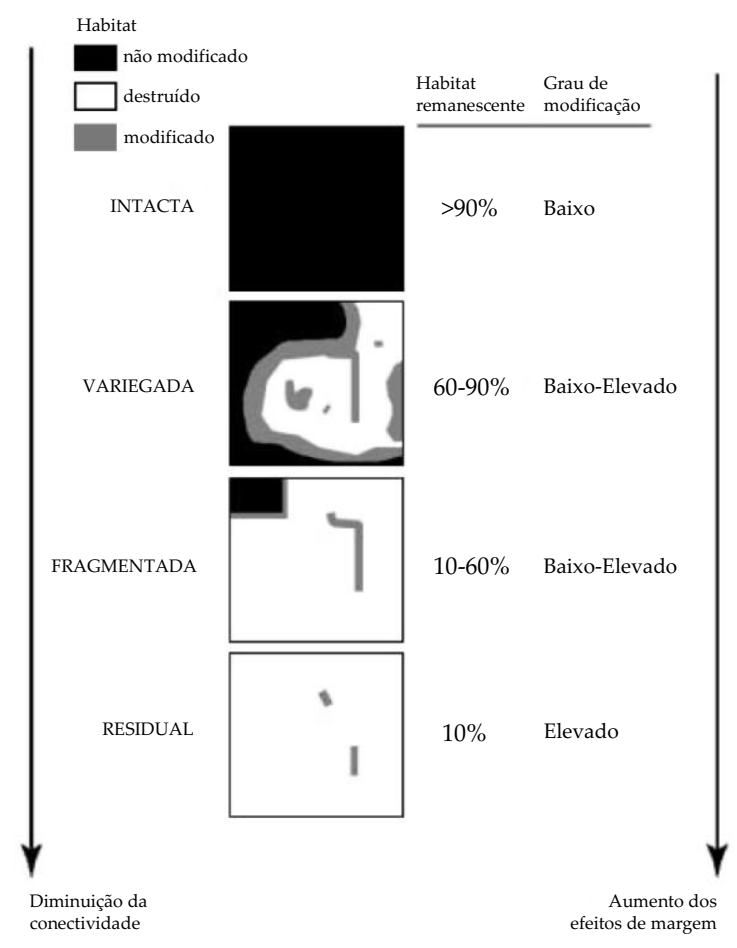


Figura 2 - Relação entre diferentes formas de perda do habitat e os padrões espaciais resultantes (adaptado de Fahrig, 2003).

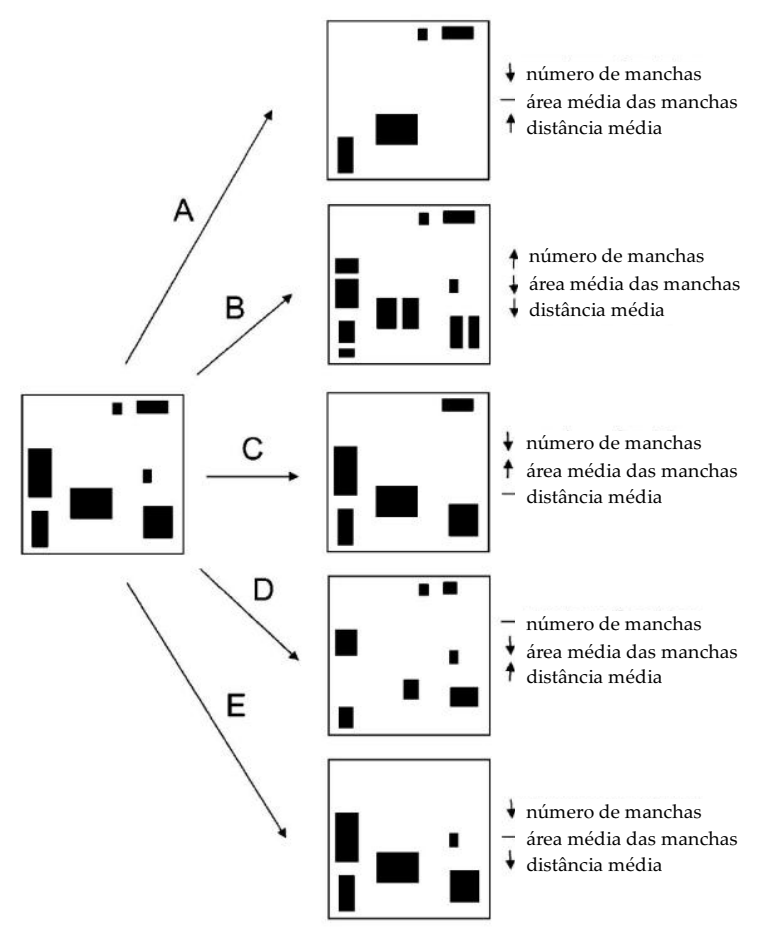

A importância dos efeitos da matriz em relação à perda da biodiversidade tem vindo a ganhar um reconhecimento acrescido; não obstante, numa revisão de estudos empíricos, datados de 1985 a 2008, Prevedello e Vieira (2010) constataram que em 95\% da investigação a influência da matriz sobre a abundância dos indivíduos de uma espécie e/ou a riqueza em espécies das comunidades nos fragmentos do habitat é bastante menor, quando comparada com os efeitos da área e do isolamento.

Fahrig (1998) examinou, através de um modelo de simulação espacial, quais as circunstâncias em que a fragmentação stricto sensu afeta, de modo significativo, a persistência da população de uma espécie virtual, independentemente da quantidade (área) e da qualidade (condições ambientais) do habitat. Para isso, realizaram-se 11 conjuntos distintos de simulações, em que se combinaram, à vez, 9 graus de fragmentação do habitat e um intervalo de valores respeitantes a 11 parâmetros que podem influenciar os efeitos da fragmentação, maximizando-os ou anulando-os. Estes parâmetros caracterizam: (i) aspetos estruturais e dinâmicos do habitat, como a área relativa, a sua efemeridade, o padrão temporal e espacial de perturbações ambientais; (ii) aspetos demográficos da população (reprodução e mortalidade); e, (iii) aspetos do 
movimento ou dispersão dos indivíduos da população, incluindo a distância máxima entre manchas do habitat por eles transponível. A partir daquelas simulações, investigou-se a relação entre cada parâmetro e o grau da fragmentação do habitat sobre o tempo de sobrevivência da população virtual. Note-se que o modelo referido partiu de um certo número de simplificações, necessárias para tornar a análise exequível, a saber: (i) representação neutra e dicotómica da paisagem (habitat vs. nonhabitat); (ii) tipo de habitat da espécie não considerado (e.g., habitat interior ou habitat da margem); (iii) efeitos de orla não considerados; (iv) movimento aleatório dos indivíduos da população; ( $v$ ) definição de uma distância máxima da dispersão; (vi) área mínima requerida pela espécie não considerada; e, (vii) relações interespecíficas não consideradas.

A principal conclusão do estudo citado é a de que a configuração espacial do habitat fragmentado apenas parece ter um efeito significativo sobre o tempo de sobrevivência da população de uma espécie, sob um número muito restrito de condições, que se devem observar simultaneamente; essas condições são as seguintes:

- o habitat da espécie cobrir menos de $20 \%$ da paisagem;

- o habitat não ser efémero (como certos habitats associados a um determinado estágio de maturação da vegetação), nem frequente e intensamente sujeito a perturbações ambientais;

- os habitats da matriz serem, no seu conjunto, inadequados à sobrevivência da espécie (observando-se aí uma elevada taxa de mortalidade), obrigando os indivíduos a dispersarem, através dela, entre as manchas remanescentes do habitat para assegurar a persistência da população no seu todo; e, por fim,

- as distâncias de dispersão serem intermédias (nem muito curtas, nem muito longas).

A última condição vem contrariar a asserção frequente de que as espécies animais com uma maior mobilidade são mais resilientes a mudanças na configuração espacial da paisagem - afirmação apoiada no argumento, originado no âmbito da modelação de metapopulações, de que uma espécie mais móvel é capaz de recolonizar com maior sucesso os fragmentos onde ocorreu a extinção local das subpopulações; no entanto, a mobilidade intrínseca que caracteriza estas espécies pode vir a tornar-se a principal 
causa do insucesso da dispersão e do escalar das extinções locais, na medida em que a fragmentação do habitat induz os indivíduos a percorrerem distâncias sucessivamente mais longas através da matriz, expondo-se assim inadvertidamente a um risco acrescido de mortalidade (cf. Fahrig, 2007). Isto significa, portanto, que a capacidade de dispersão não constitui um atributo das espécies que se possa classificar perentoriamente a priori, mas varia de modo significativo para uma mesma espécie, em função da estrutura espacial (Fahrig, 2007) e do grau de dissemelhança das condições ambientais da matriz em relação às do seu habitat (cf. Prevedello e Vieira, 2010).

No que respeita à relação entre a perda de área e a fragmentação (s.s.) do habitat, os resultados do modelo de Fahrig (1998) sugerem que a abundância relativa do habitat influencia, sobremaneira, a magnitude dos efeitos da configuração espacial sobre a persistência das subpopulações de uma espécie. Tendo em conta que a proporção do habitat varia entre $2 \%$ e 90\%, o modelo demonstrou que: (i) o efeito ecológico da fragmentação é máximo próximo dos $10 \%$ de habitat remanescente (tornando-se menos importante com o aumento e a diminuição desta percentagem); e, (ii) todas as subpopulações se extinguiram, ao se atingir uma quantidade relativa do habitat muito reduzida $(<10 \%)$, enquanto acima dos $20 \%$ todas sobreviveram. Na realidade, modelos que analisaram a variação do número de manchas, em relação à proporção do habitat existente na paisagem, concluíram consistentemente que este tende a atingir os valores máximos quando aquela se situa nos 15-20\% (Li et al., 2005), ou 15-30\% (Neel et al., 2004), correspondendo estas condições às situações de maior subdivisão do habitat, o que vem ao encontro dos resultados obtidos com as simulações supracitadas.

De acordo com Fahrig (2003), o limiar de $20-30 \%$ foi identificado em diversos modelos como sendo aquele, abaixo do qual, os efeitos da fragmentação sobre a biodiversidade se tornam proeminentes, corroborando a hipótese de que a influência da configuração espacial se subordina à quantidade do habitat existente na paisagem. Todavia, a relevância ecológica da proporção do habitat prevista nos modelos teóricos não foi, até à data, empiricamente comprovada de forma indubitável. Nomeadamente, falham as evidências quanto à existência de um "limiar de extinção", em torno dos 20\% (e.g., 
ver revisão crítica por With e King, 2001), abaixo do qual a perda de habitat será demasiado acentuada e as populações das diversas espécies se tornam incapazes de persistir, extinguindo-se. Com efeito, outros estudos sugerem que este limiar é variável entre espécies e paisagens diferentes (Ewers e Didham, 2006), dependendo tanto da sensibilidade da espécie em causa à área dos fragmentos e aos efeitos de orla (Swift e Hannon, 2010), como do facto da matriz e da configuração espacial do habitat permitirem, ou não, a dispersão entre manchas com sucesso (With e King, 2001; Swift e Hannon, 2010), estabelecendo-se, portanto, uma dinâmica de metapopulação.

A teoria da percolação examina a perda de conectividade num sistema espacialmente estruturado - sendo que, no âmbito desta teoria, conectividade se refere às ligações entre pontos que facilitam a ocorrência de fluxos, através do sistema (cf. With, 2002)-, por isso, o seu contributo tem-se revelado essencial para uma melhor compreensão da influência da distância entre manchas do habitat. No contexto da fragmentação dos habitats, é conveniente distinguir entre conectividade estrutural e conectividade funcional (With, 2002; Goodwin, 2003). A conectividade estrutural diz respeito ao grau de contiguidade do habitat, dependente da sua interrupção física na paisagem (ou da interposição de certas barreiras físicas). Já em termos funcionais, a conectividade não depende do grau de adjacência do habitat (e.g., a dispersão assistida de sementes, pólen e rizomas assegurada pelo vento, água e aves), referindo-se ao grau de inibição que os elementos estruturais da paisagem opõem aos fluxos ecológicos entre os fragmentos do habitat (Taylor et al., 1993), como no caso da matriz.

Os modelos neutros e dicotómicos (habitat vs. non-habitat) que têm na teoria da percolação a base para a modelação da dispersão de organismos (e de outros fluxos ecológicos) permitem uma mais clara avaliação da conectividade do habitat em paisagens fragmentadas (With, 1997; 2002). Segundo a autora citada, a finalidade destes modelos é a de quantificar o limiar crítico a partir do qual o habitat transita, sob a perspetiva de determinada espécie, de uma situação de conexão para um estado desconexo - o designado "limiar de percolação da paisagem" -, em função da interação entre (i) a sua capacidade de dispersão e (ii) o padrão espacial resultante da destruição (i.e., perda de área) do habitat (Fig.2). Dado que o habitat de uma espécie não tem de ser contíguo para que se considere funcionalmente conectado, o limiar de 
percolação da paisagem para essa espécie estabelecer-se-á a níveis progressivamente mais baixos de abundância relativa, na medida em que os seus organismos (indivíduos, propágulos) sejam capazes de transpor distâncias cada vez maiores entre os fragmentos, através da matriz (cf. With, 2002). No caso das plantas, cuja maioria das espécies possui uma capacidade de dispersão a curta distância (Kiviniemi e Eriksson, 1999; Walker e del Moral, 2003), o seu comportamento deverá aproximar-se do das espécies virtuais dos modelos mais restringidas pela presença de lacunas do habitat ("gap-sensitive species"; With, 2002). No seu modelo teórico, With (1999; citada em With, 2002) demonstrou que este tipo de espécies "reage" de formas distintas, em função de dois extremos no grau de fragmentação (s.s.) do habitat, quando 40-60\% deste é eliminado, nomeadamente:

- continua a dispersar com relativa facilidade, se o padrão espacial de destruição do habitat for muito disseminado - de que resulta um elevado número de fragmentos muito pequenos e pouco afastados entre si ("fragmented fractal landscapes") -, e em que a curta distância entre as manchas permite que, sob o ponto de vista da espécie, o habitat continue a funcionar como conectado;

- passa a dispersar com grande dificuldade, quando extensas áreas do habitat são destruídas em bloco, originando um padrão espacial de destruição aglomerado, que abre grandes lacunas (i.e., áreas de non-habitat) - e interpõe longas distâncias entre os fragmentos que restam em número reduzido, mas mantendo uma dimensão maior ("clumped fractal landscapes") -, difíceis de ultrapassar, pelo que, para a espécie, o habitat deixa de funcionar como conectado.

Nesta última situação, a maior distância entre as manchas inibe as recolonizações, através dos mecanismos de dispersão, determinando que cada subpopulação da espécie permaneça efetivamente confinada e isolada das restantes, razão pela qual pode vir a tornar-se vulnerável à extinção local por processos estocásticos. Por outro lado, no primeiro caso, apesar de a espécie ser capaz de dispersar entre os numerosos fragmentos remanescentes, a grande maioria deles tem uma superfície reduzida, que pode ser muito inferior à área mínima requerida pela espécie (With, 1999; citada em With, 2002) para manter uma população com um tamanho mínimo viável; desta 
maneira, a conectividade do habitat não garante, por si só, a persistência da espécie (With, 2002).

Resumindo, a análise da conectividade do habitat, em modelos desenvolvidos com base na teoria da percolação, permite concluir que:

- a perda de conectividade pode ocorrer na sequência da perda de área do habitat, mas é sobretudo ditada pela inter-relação da configuração espacial resultante com a capacidade de dispersão das espécies envolvidas; e,

- a conectividade do habitat em paisagens fragmentadas, próximas do limiar de percolação, não constitui condição suficiente para a persistência de uma espécie, se as áreas das manchas conectadas forem demasiado pequenas para assegurar a viabilidade das suas subpopulações.

\section{Conclusão}

A abordagem espacial desenvolvida pela Ecologia da Paisagem sobre as temáticas que sempre interessaram à Biogeografia, levaria a crer que ambas as áreas científicas contribuiriam de forma assídua e sinergística para o avanço comum da investigação ecológica e biogeográfica. Contudo, tanto a análise espacial como o quadro teórico da Ecologia da Paisagem - que se foram consolidando nos últimos 25-30 anos - têm sido muito menos explorados no âmbito da Biogeografia do que no da Biologia e da Ecologia (Kent, 2007; 2009). Numa notável reflexão, Martin Kent (2003) aponta como razões pelas quais a dimensão espacial foi, a partir dos anos 1950, relegada para segundo plano em Geografia Física, o advento do conceito de "ambiente" que se veio substituir ao de espaço (conotado com uma abordagem descritiva, ao passo que o primeiro se associou à síntese e interpretação), a ênfase dada às modificações ambientais, à compreensão da sua dinâmica temporal e dos processos que as determinam. Este foco no tempo e nos processos fica bem ilustrado na abordagem metodológica, comum à Biogeografia e à Geomorfologia, em que diferentes áreas no espaço são tomadas como representativas de diferentes fases no tempo (e.g., análise dos processos da sucessão ecológica e da evolução das formas de relevo) - o espaço como substituto do tempo. Ressalve-se, porém, que a crescente utilização dos 
sistemas de informação geográfica, da fotografia aérea e de imagens de satélite impulsionou a investigação recente, em torno da análise de padrões espaciais, no âmbito da Geomorfologia Dinâmica e da Climatologia Urbana, domínios que, por isso mesmo, se encontram conceptual e metodologicamente mais próximos da Ecologia da Paisagem.

A interpretação biogeográfica da distribuição espacial de espécies e comunidades biológicas apoia-se recorrentemente nas condições abióticas e relações interespecíficas, à escala local, e/ou nos padrões à escala regional (cf. Dupré e Ehrlén, 2002), sem consideração explícita da dimensão espacial (Hanski, 1999; citada em Kent, 2003). Por sua vez, a teoria da metapopulação atesta a importância da configuração espacial do habitat, a uma escala intermédia. Ora, fatores explicativos a diferentes escalas espaciais não se excluem mutuamente, pelo que uns não podem ser entendidos como a negação dos outros; pelo contrário, tal como Hanski (1999, p. 261; citada em Kent, 2003) afirma: "The novelty of spatial ecology is in the claim that the spatial locations of individuals, populations and communities can have [...] significant consequences on dynamics as birth and death rates, competition and predation". Um exemplo da desejada integração destes fatores explicativos, através de um modelo de análise espacial, é o do trabalho desenvolvido por Gardner e Engelhardt (2008), relativo a comunidades de plantas.

Por fim, a síntese apresentada neste artigo, e que não é de forma alguma exaustiva, pretendeu mostrar o interesse e a oportunidade dos estudos empíricos que examinem o padrão de distribuição espacial de plantas e animais no contexto da dinâmica de metapopulações (e metacomunidades) - de maneira a reunir evidências suficientemente consistentes que confirmem ou rejeitem a teoria -, tendo em conta que muitos habitats que são objeto do estudo biogeográfico mostram um grau maior ou menor de fragmentação (e.g., Laranjeira, 2010). Esta proposta assoma-se, sem dúvida, como um campo fértil para a pesquisa futura em Biogeografia. 


\section{Referências bibliográficas}

Arrhenius, O. (1921). "Species and area". Journal of Ecology 9: 95-99.

August, P.; Iverson, L.; Nugranad, J. (2002). Human conversion of terrestrial habitats. Applying Landscape Ecology in Biological Conservation. K. Gutzwiller (Ed.). New York, Springer-Verlag: 198-224.

Bender, D.J.; Tischendorf, L.; Fahrig, L. (2003). "Using patch isolation metrics to predict animal movement in binary landscapes". Landscape Ecology 18:17-39.

Bonan, G.B. (2002). Ecological Climatology: Concepts and Applications. Cambridge, Cambridge University Press, 690p.

Brown, J.H.; Kodric-Brown, A. (1977). "Turnover rates in insular biogeography: effect of immigration on extinction". Ecology 58:445-449.

Collinge, S.K. (1996). "Ecological consequences of habitat fragmentation: implications for landscape architecture and planning". Landscape and Urban Planning 36: 59-77.

Cowell, C.M.; Parker, A.J. (2004). "Biogeography in the Annals". Annals of the Association of American Geographers 94:256-268.

Denslow, J.S. (1985). Disturbance-mediated coexistence of species. The Ecology of Natural Disturbance and Patch Dynamics. S.T.A. Pickett e P.S. White (Eds.). New York, Academic Press:307-323.

Dias, B. (2001). Demandas governamentais para o monitoramento da diversidade biológica brasileira. Conservação da Biodiversidade em Ecossistemas Tropicais: Avanços Conceituais e Revisão de Novas Metodologias de Avaliação e Monitoramento. I. Garay e B. Dias (Eds.). Petrópolis, Editora Vozes:17-28.

Dupré, C., Ehrlén, J. (2002). "Habitat configuration, species traits and plant distributions". Journal of Ecology 90: 796-805.

Ewers, R.M.; Didham, R.K. (2006). "Confounding factors in the detection of species responses to habitat fragmentation". Biological Reviews 81:117-142. 
Fahrig, L. (1998). "When does fragmentation of breeding habitat affect population survival?". Ecological Modelling 105:273-292.

Fahrig, L. (2003). "Effects of habitat fragmentation on biodiversity". Annual Review of Ecology, Evolution, and Systematics 34:487-515.

Fahrig, L. (2005). When is a landscape perspective important?. Issues and Perspectives in Landscape Ecology. J. Wiens e M. Moss (Eds.). Cambridge, Cambridge University Press: $3-10$.

Fahrig, L. (2007). "Non-optimal animal movement in human-altered landscapes". Functional Ecology 21(6): 1003-1015.

Feinsinger, P. (2001). Designing Field Studies for Biodiversity Conservation, Washington, Island Press (The Nature Conservancy).

Fischer, J.; Lindenmayer, D.B. (2007). "Landscape modification and habitat fragmentation: a synthesis". Global Ecology and Biogeography 16:265-280.

Forman, R.T.T. (1995). Land Mosaics: The Ecology of Landscapes and Regions. Cambridge, Cambridge University Press.

Fortin, M.-J.; Boots, B.; Csillag, F.; Remmel, T.K. (2003). "On the role of spatial stochastic models in understanding landscape indices in ecology". Oikos 102:203-212.

Gardner, R.; Engelhardt, K. (2008). "Spatial processes that maintain biodiversity in plant communities". Perspectives in Plant Ecology, Evolution and Systematics 9: 211228

Goodwin, B.J. (2003). "Is landscape connectivity a dependent or independent variable?". Landscape Ecology 18:687-699.

Gustafson, E.J. (1998). “Quantifying landscape spatial pattern: What is the state of the art?". Ecosystems 1:143-156.

Gustafson, E.J.; Diaz, N. (2002). “Landscape pattern, timber extraction, and biological conservation". Applying Landscape Ecology in Biological Conservation. K. Gutzwiller (Ed.), New York, Springer-Verlag: 244-265.

Hanski, I.A. (1999). Metapopulation Ecology. Oxford, Oxford University Press. 
Hanski, I.A.; Gilpin, M.E. (Eds.) (1997). Metapopulation Biology: Ecology, Genetics, and Evolution. San Diego, Academic Press.

Henle, K.; Davies, K.F.; Kleyer, M.; Margules, C.; Settele, J. (2004). "Predictors of species sensitivity to fragmentation". Biodiversity and Conservation 13:207-251.

Hubbell, S.P. (2001). The Unified Neutral Theory of Biodiversity and Biogeography. Princeton e Oxford, Princeton University Press.

Huston, M.A. (1994). Biological Diversity: The Coexistence of Species on Changing Landscapes. Cambridge, Cambridge University Press.

Janzen, D.H. (1986). The eternal external threat. Conservation Biology: The Science of Scarcity and Diversity. M.E. Soulé (Ed.). Sunderland, Sinauer Associates:286-303.

Jentsch, A. (2007). "The challenge to restore processes in face of nonlinear dynamics: on the crucial role of disturbance regimes". Restoration Ecology 15: 334-339.

Karr, J.R. (1997). Measuring biological integrity. Principles of Conservation Biology. G.K. Meffe, C.R. Carroll e Contrib., Sunderland, Sinauer Associates: 483-485.

Kent, M. (2003). Space: making room for space in physical geography. Key Cooncepts in Geography. S. Holloway; S. Rice; G. Valentine (Eds.). London, SAGE Publications.

Kent, M. (2007). "Biogeography and landscape ecology". Progress in Physical Geography 31(3): 345-355.

Kent, M. (2009). "Biogeography and landscape ecology: the way forward - gradients and graph theory". Progress in Physical Geography 33(3): 424-436.

Kiviniemi, K.; Eriksson, O. (1999). "Dispersal, recruitment and site occupancy of grassland plants in fragmented habitats". Oikos 86:241-253.

Krebs, C.J. (2001). Ecology: The Experimental Analysis of Distribution and Abundance, San Francisco, Benjamin Cummings.

Laranjeira, M.M. (2010). Fragmentação pelo Pisoteio dos Sistemas de Duna Frontal: Casos de Vila Nova de Gaia. Doutoramento, Universidade do Minho.

Laurance, W.F.; Camargo, J.; Luizão, R.; Laurance, S.; Pimm, S.; Bruna, E.; Stouffer, P.; Williamson, G.; Benítez-Malvido, J.; Vasconcelos, H.; Van Houtan, K.; Zartman, C.; 
Boyle, S.; Didham, R.; Andrade, A.; Lovejoy, T. (2011). "The fate of Amazonian forest fragments: a 32-year investigation". Biological Conservation 144(1): 56-67

Laurance, W.F.; Lovejoy, T.; Vasconcelos, H.; Bruna, E.; Didham, R.; Stouffer, P.; Gascon, C.; Bierregaard, R.; Laurance, S.; Sampaio, E. (2002). "Ecosystem decay of Amazonian forest fragments: a 22-year investigation". Conservation Biology 16:605618.

Leibold, M.A.; Holyoak, M.; Mouquet, N.; Amarasekare, P.; Chase, J.; Hoopes, M.; Holt, R.; Shurin, J.; Law, R.; Tilman, D.; Loreau, M.; Gonzalez, A. (2004). "The metacommunity concept: a framework for multi-scale community ecology". Ecology Letters, 7:601-613.

Levins, R. (1969). "Some demographic and genetic consequences of environmental heterogeneity for biological control". Bulletin of Entomological Society of America 15: 237-240.

Li, H.; Wu, J. (2004). "Use and misuse of landscape indices". Landscape Ecology 19: 389-399.

Li, X.; He, H.S.; Bu, R.; Wen, Q.; Chang, Y.; Hu, Y.; Li, Y. (2005). "The adequacy of different landscape metrics for various landscape patterns". Pattern Recognition 38: 2626-2638.

Łaska, G. (2001). "The disturbance and vegetation dynamics: a review and an alternative framework". Plant Ecology 157: 77-99.

MacArthur, R.H.; Wilson, E.O. (1967). The Theory of Island Biogeography. Princeton, Princeton University Press.

Matthews, J.A. (1996). "Gleason, H.A. 1939: The individualistic concept of the plant association. The American Midland Naturalist 21, 92-110 (Classics in physical geography revisited)". Progress in Physical Geography 20: 193-203.

Mclntyre, S.; Hobbs, R. (1999). "A framework for conceptualizing human effects on landscapes and its relevance to management and research models". Conservation Biology 13: 1282-1292. 
Meffe, G.K.; Carroll, C.R. e Contrib. (1997). Principles of Conservation Biology. Sunderland, Sinauer Associates.

Neel, M.C.; McGarigal, K.; Cushman, S.A. (2004). "Behavior of class-level landscape metrics across gradients of class aggregation and area". Landscape Ecology 19: 435455.

Noble, I.R.; Slatyer, R.O. (1980). "The use of vital attributes to predict successional changes in plant-communities subject to recurrent disturbances". Vegetatio 43:5-21.

Olff, H.; Ritchie, M.E. (2002). "Fragmented nature: consequences for biodiversity". Landscape and Urban Planning 58:83-92.

Perry, G.L.W. (2002). "Landscapes, space and equilibrium: shifting viewpoints". Progress in Physical Geography 26:339-359.

Pité, M.T.R., Avelar, T. (1996). Ecologia das Populações e das Comunidades: Uma Abordagem Evolutiva do Estudo da Biodiversidade. Lisboa, Fundação Calouste Gulbenkian.

Prevedello, J.A.; Vieira, M.V. (2010), "Does the type of matrix matter? A quantitative review of the evidence". Biodiversity and Conservation 19(5): 1205-1223.

Rohde, K. (2005). Nonequilibrium Ecology. Cambridge, Cambridge University Press, $236 p$.

Smith, A.C.; Koper, N.; Francis, C.M.; Fahrig, L. (2009). "Confronting collinearity: comparing methods for disentangling the effects of habitat loss and fragmentation". Landscape Ecology 24(10): 1271-1285.

Swift, T.L.; Hannon, S.J. (2010). "Critical thresholds associated with habitat loss: a review of the concepts, evidence, and applications". Biological Reviews 85(1): 35-53.

Taylor, P.D.; Fahrig, L.; Henein, K.; Merriam, G. (1993). “Connectivity is a vital element of landscape structure". Oikos 68: 571-573.

Thomas, C.D.; Kunin, W.E. (1999). "The spatial structure of populations". Journal of Animal Ecology 68: 647-657. 
Turner, M.G. (1989). "Landscape ecology: the effect of pattern on process". Annual Review of Ecology and Systematics 20: 171-197.

Turner, M.G. (2005). "Landscape ecology: What is the state of the science?". Annual Review of Ecology, Evolution, and Systematics 36: 319-344

Walker, L.R.; del Moral, R. (2003). Primary Succession and Ecosystem Rehabilitation. Cambridge, Cambridge University Press.

White, P.; Pickett, S. (1985). Natural disturbance and patch dynamics: an introduction. The Ecology of Natural Disturbance and Patch Dynamics. S. Pickett e P. White (Eds.). New York, Academic Press: 3-13.

Wiens, J.A. (2002). Central concepts and issues of landscape ecology. Applying Landscape Ecology in Biological Conservation. K. Gutzwiller (Ed.), New York, SpringerVerlag: 3-21.

With, K.A. (1997). "The application of neutral landscape models in conservation biology". Conservation Biology 11: 1069-1080.

With, K.A. (1999). "Is landscape connectivity necessary and sufficient for wildlife management?". Forest Fragmentation: Wildlife and Management Implications. J.A. Rochelle; L.L. Lehmann; J. Wisniewski (Eds.), Leiden, Brill Academic Publishers: 97-115.

With, K.A. (2002). Using percolation theory to assess landscape connectivity and effects of habitat fragmentation. Applying Landscape Ecology in Biological Conservation. K. Gutzwiller (Ed.), New York, Springer-Verlag: 105-130.

With, K.A.; King, A.W. (2001). "Analysis of landscape sources and sinks: the effect of spatial pattern on avian demography". Biological Conservation 100: 75-88.

Woodley, S.; Theberge, J. (1992). Monitoring for ecosystem integrity in Canadian national parks. Science and the Management of Protected Areas. J.H.M. Willison; S. Bondrup-Nielsen; C. Drysdale; T.B. Herman; N.W.P. Munro; T.L. Pollock (Eds.), Amsterdam, Elsevier Science: 369-377.

Wu, J.; Hobbs, R. (2002). "Key issues and research priorities in landscape ecology: an idiosyncratic synthesis". Landscape Ecology 17: 355-365. 
